\title{
ROTULAGEM DE ALIMENTOS COMO VEÍCULO DE INFORMAÇÃO AO CONSUMIDOR: ADEQUAÇÕES E IRREGULARIDADES
}

\author{
NÁDIA YOSHIZAWA * \\ RENATA TOEDTER POSPISSIL * \\ ALESSANDRA GODINHO VALENTIM * \\ DANIELA SEIXAS * \\ FERNANDA SANT'ANNA ALVES * \\ FRANCIELE CASSOU * \\ ILKA YOSHIDA * \\ ROSE APARECIDA SEGA ** \\ LYS MARY BILESKI CÂNDIDO ***
}

\begin{abstract}
Investigou-se a adequação de 220 rótulos de alimentos de diferentes categorias com ênfase nos alimentos para fins especiais e os adicionados de nutrientes essenciais. Observou-se que a maioria dos fabricantes não cumpre a legislação brasileira em vigor, pois todos os rótulos analisados apresentaram alguma irregularidade. Verificou-se ausência de informações sobre o modo de conservação do produto $(25 \%)$ e sobre a presença de corante ou aroma artificial $(16,77 \%)$ nos alimentos. Também foram encontradas informações que poderiam causar engano ao consumidor $(19,9 \%)$, incluindo alegações de propriedades terapêuticas dos alimentos. Em mais de $50 \%$ dos rótulos analisados, segundo a Resolução RDC 40/01, foram constatadas incorreções relativas à informação nutricional. As principais discordâncias em relação às demais portarias vigentes envolveram informações e declarações obrigatórias e específicas de cada norma. Concluiu-se que a maioria dos rótulos dos produtos analisados encontrava-se em desacordo com a legislação, havendo necessidade de fiscalização mais eficaz para reforçar o compromisso dos fabricantes com o consumidor.
\end{abstract}

PALAVRAS-CHAVE: ALIMENTOS-ROTULAGEM; ALIMENTOS-LEGISLAÇÃO.

* Bolsistas de Extensão, Alunos do Curso de Nutrição, Universidade Federal do Paraná (UFPR), Curitiba, PR. (e-mail: nadiayoshizawa@hotmail.com).

** Médica Veterinária, Secretaria de Saúde do Estado do Paraná, Curitiba, PR. (email: rose_sega@yahoo.com.br).

*** Farmacêutica Industrial, Laboratório de Pesquisa e Pós-Graduação, Departamento de Nutrição, UFPR, Curitiba, PR. (e-mail: lysmary@ufpr.br). 


\section{INTRODUÇÃO}

A busca pela qualidade de vida e a diversidade de alimentos industrializados existentes no mercado têm tornado o consumidor cada vez mais exigente e preocupado com a segurança alimentar. De acordo com o item 3, do artigo 6ª da Lei 8078/90 (Código de Proteção e Defesa do Consumidor) é por meio do rótulo dos alimentos que se tem acesso a informações como quantidade, características nutricionais, composição, qualidade e riscos que os produtos poderiam apresentar (1).

Os regulamentos técnicos brasileiros, editados a partir de janeiro de 1998, procuraram incorporar conceitos da comunidade científica internacional, tendo como base as recomendações das comissões do Codex Alimentarius. Tais normas adotam critérios hierárquicos, partindo do geral (como a Resolução RDC 259/02 (2) que substituiu a Portaria SVS/MS n.42/9 (8) sobre Rotulagem Geral de Alimentos Embalados) para o específico (Figura 1). No segundo nível estão a Resolução RDC 40/01 (4) sobre Rotulagem Nutricional e a Portaria SVS/MS 27/98 (5) referente à Informação Nutricional Complementar. Cinco grupos de alimentos, regulamentados por Portarias específicas formam o terceiro nível. São os Alimentos para Fins Especiais - ALIFINS, Portaria SVS/ MS n.29/98 (6), os Alimentos para Controle de Peso (7), os Alimentos Adicionados de Nutrientes Essenciais, Portaria SVS/MS n.31/98 (8), os Suplementos Vitamínicos e Minerais, Portaria SVS/MS n.32/98 (9), os Adoçantes de Mesa e Adoçantes Dietéticos, Portaria SVS/ MS n.38/98 (10), e Sal Hipossódico, Portaria SVS/MS n.54/95 (11).

Os alimentos para fins especiais (6) incluem aqueles para dietas com restrição de nutrientes, para ingestão controlada de nutrientes e os destinados a grupos populacionais específicos. No segundo grupo referido encontram-se os alimentos para controle de peso (7), alimentos para praticantes de atividade física (12) e alimentos para nutrição enteral (13). O terceiro grupo é composto pelos alimentos de transição para lactentes e crianças de primeira infância (14), os alimentos à base de cereais para alimentação infantil (15), as fórmulas infantis e de seguimento (16) e os alimentos para gestantes e nutrizes (17). A Resolução RDC n.222/02 regulamenta a promoção comercial de alimentos para lactentes e crianças de primeira infância (18).

É necessário que fabricantes de alimentos assegurem aos consumidores o acesso a informações úteis e confiáveis sobre o produto que estão 
adquirindo. Para conquistar a confiança do cliente, os fabricantes devem atender às exigências legais dos regulamentos técnicos de rotulagem de alimentos.

Este trabalho teve por objetivo investigar a adequação dos rótulos de diferentes produtos alimentícios, nacionais e importados, ao disposto na legislação brasileira vigente. Seu desenvolvimento resultou da parceria estabelecida entre a Universidade Federal do Paraná (UFPR), pelo Departamento de Nutrição e pela Pró-Reitoria de Extensão e Cultura, e o Instituto de Saúde do Paraná da Secretaria Estadual de Saúde (SESAPR).

\section{METODOLOGIA}

Foram analisados 220 rótulos de diversos produtos alimentícios no período de outubro de 2001 a fevereiro de 2002. As informações de rotulagem foram coletadas em supermercados, farmácias e lojas de produtos especializados. Para a análise dos rótulos foi utilizada Ficha de Avaliação de Rotulagem desenvolvida pelos participantes do trabalho para servir de suporte aos profissionais da Vigilância Sanitária. Verificou-se a concordância das declarações de rotulagem observadas nos alimentos com as recomendações de cada regulamento técnico.

Os produtos alimentícios foram selecionados aleatoriamente, sendo analisado um exemplar de cada marca, independentemente de outras formas produzidas (sabores ou embalagens com conteúdo líquido diferentes). As informações dos rótulos foram confrontadas com a Resolução SVS/MS n.42/98 (3), em vigor durante a realização do levantamento e depois substituída pela Resolução RDC ANVISA/MS n.259/ 02 (2), e com a Resolução ANVISA/MS RDC n.40/01 (4). Os rótulos dos alimentos foram divididos em categorias, conforme as respectivas Portarias. Assim, 113 produtos corresponderam a alimentos para fins especiais (6), 29 a alimentos adicionados de nutrientes essenciais (8), 19 a alimentos de transição para lactentes e crianças de primeira infância, alimentos à base de cereais para alimentação infantil, fórmulas infantis para lactentes e alimentos para lactentes e crianças de primeira infância $(14,15,16,18), 16$ a adoçantes de mesa (10), 13 a alimentos para praticantes de atividade física (12), 6 a alimentos para controle de peso (7), 2 a sal hipossódico (11), 1 a suplemento vitamínico e mineral (9), 1 a complemento alimentar para gestantes e nutrizes (17) e 20 pertencentes à categoria dos novos alimentos $(19,20)$. 


\section{FIGURA 1 - NORMAS BRASILEIRAS PARA ALIMENTOS}

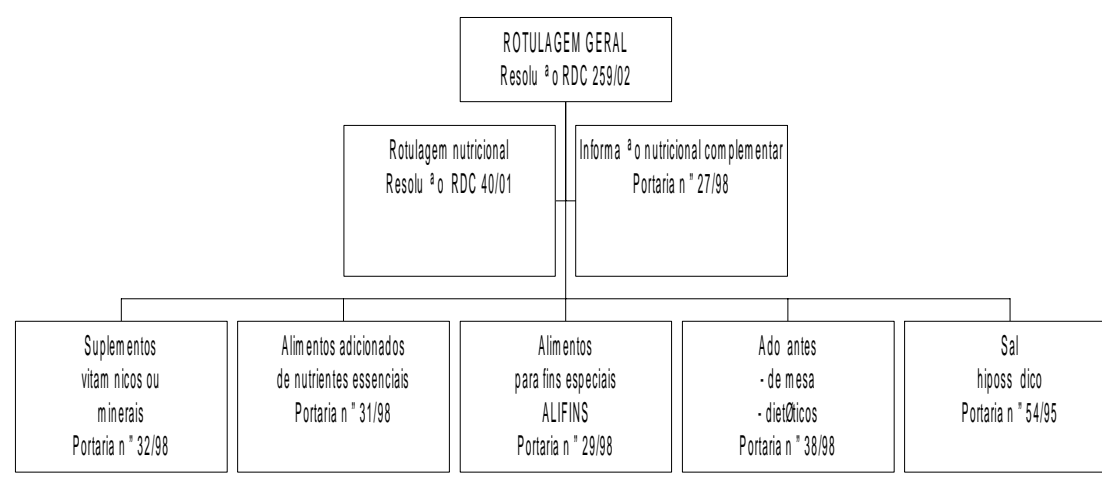

Fonte: Adaptado de CÂNDIDO, 2000 (21). 


\section{RESULTADOS E DISCUSSÃO}

Os rótulos de todos os produtos alimentícios comercializados devem atender à Resolução RDC ANVISA/MS 259/02 (Portaria SVS/MS 42/98, quando o levantamento foi efetuado) (2, 3) e à Resolução RDC 40/01 (4). Entretanto, nenhum dos 220 rótulos analisados encontrava-se totalmente de acordo com esses regulamentos técnicos. Não é permitido o uso de vocábulos, sinais, denominações, símbolos, emblemas, ou ilustrações que levem o consumidor à informação falsa, incorreta, insuficiente, ou que possa induzi-lo a equívoco, confusão ou engano, em relação à verdadeira natureza, composição, procedência, tipo, qualidade, quantidade, rendimento ou forma de uso do alimento.

Conforme mostra a Figura 2, em $25 \%$ dos rótulos não constavam o modo de conservação do produto e 19,9\% continham informações que poderiam induzir o consumidor a erro. Foram encontradas expressões como "pode repetir que é light", "não engorda", "tem fibras que inibem o apetite" ou "contém ingredientes que ativam as proteínas", bem como alegações incorretas de isenção de gordura. Além disso, 16,77\% dos rótulos não apresentavam indicação de corante ou de aroma artificial e em 11,81\% não constavam a expressão "conteúdo líquido" anterior à declaração do conteúdo do produto. A declaração da natureza do produto foi omitida em $99,55 \%$ dos rótulos analisados, a qual era obrigatória pela Portaria em vigor na época da pesquisa (3). Tal declaração foi revista pela ANVISA, não sendo mais exigida pela RDC 259/02 (2).

\section{FIGURA 2- ADEQUAÇÃO DE ROTULAGEM SEGUNDO A RESOLUÇÃOO RDC ANVS/MS 259/02}

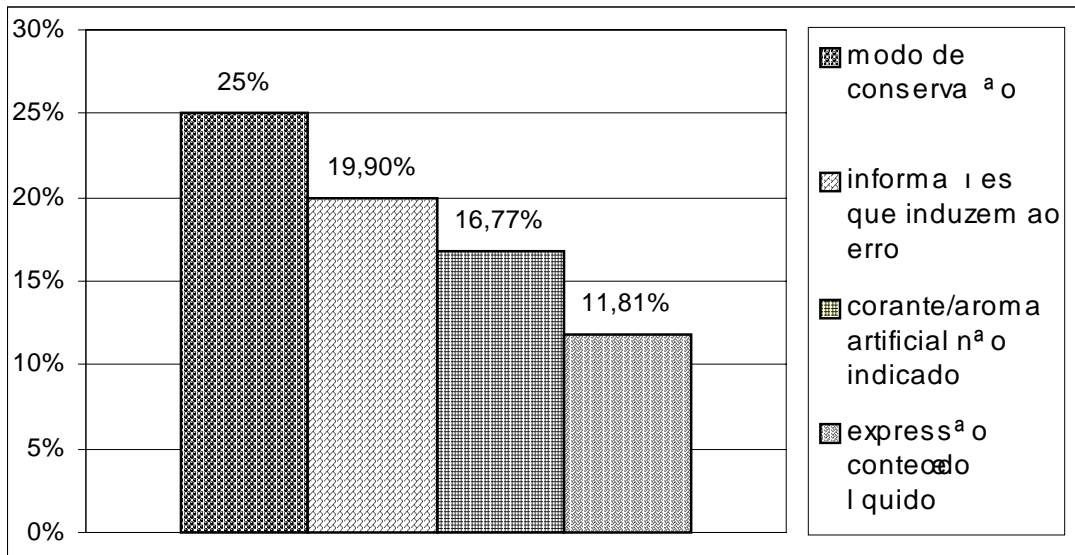


Com relação à Resolução RDC n.40/01 (Figura 3), mais de 50\% dos rótulos analisados continham informação nutricional incorreta (expressa por porção em ordem incorreta e por 100 gramas) ou não a apresentavam quando se tratava da declaração simplificada (4). Aproximadamente, $60 \%$ dos rótulos dos produtos não indicavam a porcentagem dos valores diários recomendados (\%VD) por porção e $40 \%$ não continham informação sobre o peso do produto por porção.

\section{FIGURA 3 - IRREGULARIDADES RELACIONADAS À RESOLUÇÃO RDC n.40/01}

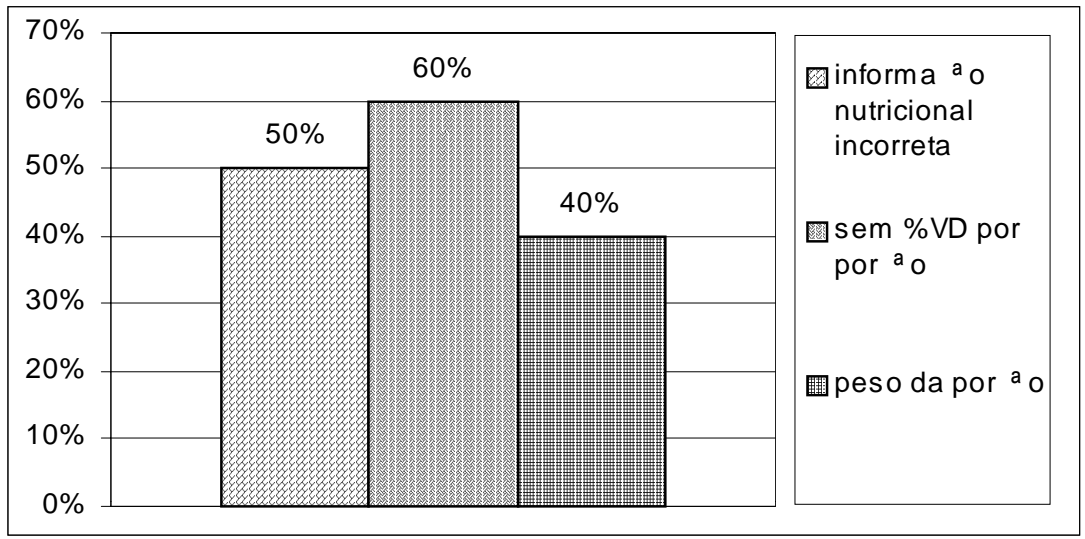

$\% \mathrm{VD}=$ Porcentagem dos valores diários recomendados.

Alimentos para fins especiais são formulados ou processados com modificações no conteúdo de nutrientes, visando utilização em dietas diferenciadas (e/ou opcionais) para atender às necessidades de pessoas em condições metabólicas e fisiológicas específicas (Portaria SVS/MS 29/98) (6). A análise dos rótulos desse grupo de alimentos mostrou ausência de justificativa para o uso da denominação light $(9,09 \%)$ e de designação e finalidade do produto (30\%). Quanto às advertências em destaque e em negrito, $20 \%$ dos rótulos não apresentavam advertência para diabéticos (contêm mono e dissacarídios). Em 23\% dos rótulos dos produtos contendo polióis não foi observada a advertência quanto ao efeito laxativo e $50 \%$ dos rótulos não realçavam a importância do alimento ser consumido sob orientação de médico ou nutricionista (Figura 4). 


\section{FIGURA 4 - ADEQUAÇÃO DE ROTULAGEM DE ALIMENTOS PARA}

FINS ESPECIAIS

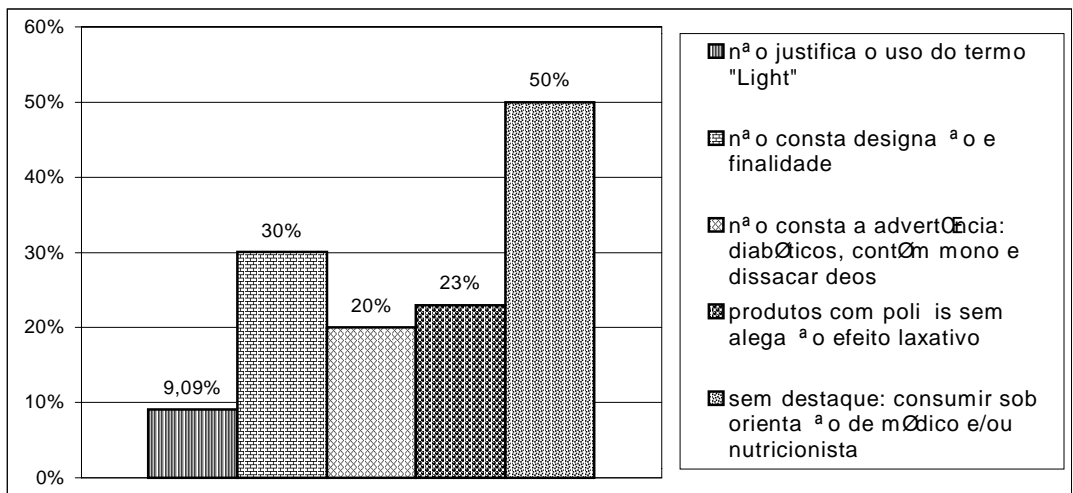

Dentre os rótulos dos alimentos para controle de peso (Portaria SVS/MS 30/98) (7), 50\% não apresentavam advertência para diabéticos que o produto continha mono e dissacarídios, $16,67 \%$ não declaravam que se deve aumentar a ingestão de água ao consumi-los e em 16,67\% essa informação não estava destacada. A advertência relacionada ao consumo sob indicação de médico ou nutricionista apareceu em $66,67 \%$ dos rótulos, mas em 33,33\% não havia informação nutricional por $100 \mathrm{~g}$ ou $100 \mathrm{~mL}$ e por porção, nem indicavam o número de porções diárias a serem consumidas.

Quanto ao item suplementos vitamínicos e minerais (Portaria SVS/MS 32/98) (9), o produto analisado não apresentou advertências específicas em destaque. O mesmo ocorreu em $15,4 \%$ dos rótulos de alimentos destinados aos praticantes de atividade física (Portaria SVS/MS 222/98) (12).

Em $57,14 \%$ dos rótulos de alimentos infantis à base de cereais (Portaria SVS/MS 36/98) (16) não constavam a designação que caracteriza o produto (3 indicados para crianças, adolescentes e adultos) e $28,57 \%$ não continham a advertência de que o produto não deve ser utilizado na alimentação dos lactentes nos primeiros nove meses de vida.

Com relação aos sucedâneos do leite materno (Resolução RDC 222/02) (18), os rótulos não apresentavam as seguintes advertências: quanto ao uso na alimentação de lactente nos primeiros 6 meses de vida $(60 \%)$, 
que o aleitamento materno deve ser mantido após introdução de alimentos sólidos (80\%) e que a criança amamentada ao seio não necessita de mamadeira ou bico (100\%).

No caso dos alimentos adicionados de nutrientes essenciais (Portaria SVS/MS 31/98) (8), 75,86\% dos rótulos não informavam a composição nutricional em termos de porcentagem de ingestão diária recomendada (\% IDR) por $100 \mathrm{~g}$ ou por $100 \mathrm{~mL}$ ou número de porções.

Em relação aos adoçantes de mesa (Portaria SVS/MS 38/98) (10), 6,67\% dos produtos contendo aspartame não apresentavam em destaque a expressão "contém fenilalanina".

Quanto ao sal hipossódico (Portaria n.54/95) (11), 50\% dos produtos não mencionavam o teor de sódio e potássio por $100 \mathrm{~g}$ ou por porção, nem o número de porções diárias a serem ingeridas.

No mercado internacional observa-se tendência pela valorização de produtos alimentícios que produzem benefícios específicos à saúde, além do conteúdo de nutrientes tradicionais. Tais alimentos aportam níveis significativos de componentes biologicamente ativos, sendo chamados de funcionais, nutracêuticos ou por outras denominações. São vistos como promotores de saúde e podem estar relacionados à redução de riscos de certas doenças. Essa categoria de alimentos não é contemplada pela legislação brasileira, contudo são aceitas declarações de propriedades funcionais ou de saúde nos rótulos dos alimentos conforme as Resoluções ANVS/MS n.16/99 e n.18/99 (19, 20). Novos alimentos e/ou novos ingredientes são os alimentos ou substâncias sem histórico de consumo no país, ou alimentos com substâncias já consumidas, e que, entretanto, venham a ser adicionadas ou utilizadas em níveis muito superiores aos normalmente observados na dieta regular (19). A Resolução ANVS/MS n.18/99 (20) considera a alegação de propriedade funcional como o papel metabólico ou fisiológico que o nutriente ou não-nutriente exerce no crescimento, desenvolvimento, manutenção e outras funções normais do organismo humano. A alegação de propriedade de saúde implica na existência de relação entre o alimento ou ingrediente com doença ou condição relacionada à saúde. Segundo essa norma não são permitidas alegações de saúde que façam referência à cura ou prevenção de doenças. Para este mercado crescente, as principais recomendações são: declarações que possam ser entendidas pelo consumidor, indicação de uso dos produtos, credibilidade, harmonização da legislação internacional com definição comum dos termos; receptibilidade a métodos alternativos, 
prevenção do risco de declarações inadequadas ou enganosas, integração com universidades e entidades de pesquisa para validação das alegações de rotulagem, avaliação custo/benefício e criatividade para a rotulagem.

A análise de rótulos da categoria Novos Alimentos (Figura 5) revelou que $20 \%$ dos produtos não detinham registro no Ministério da Saúde, apresentando apenas o número de protocolo. Além disso, 10\% alegava tratar-se de produto $100 \%$ natural e somente $45 \%$ obedeciam às exigências da Resolução SVS/MS18/99. Foram encontradas indicações como: "... fornece elementos necessários à formação do colágeno que vai promover o enrijecimento dos tecidos e da pele, prevenindo a flacidez e o envelhecimento precoce, sendo também utilizado como coadjuvante no combate ao peso"; "combate artrose, artrite, dor nas juntas, bursite, bico de papagaio, dor na coluna"; "... colaboram na proteção ao sistema cardiovascular, ... ajudam a reduzir os níveis de colesterol no sangue"; "o consumo de produto com ômega 3 e ômega 6 faz bem à saúde", além de informações que indicam prevenção e tratamento de doenças. Os 20 produtos analisados nessa categoria também evidenciaram irregularidades relacionadas à rotulagem nutricional. Não apresentavam informação nutricional por porção (50\%) ou a apresentavam por $100 \mathrm{~g}(15 \%)$ ou em ordem incorreta (5\%). Também não expressavam a quantidade em porcentagem do valor diário (70\%) nem o peso da porção (30\%).

\section{FIGURA 5 - IRREGULARIDADES ENCONTRADAS NOS PRODUTOS DA CATEGORIA NOVOS ALIMENTOS}

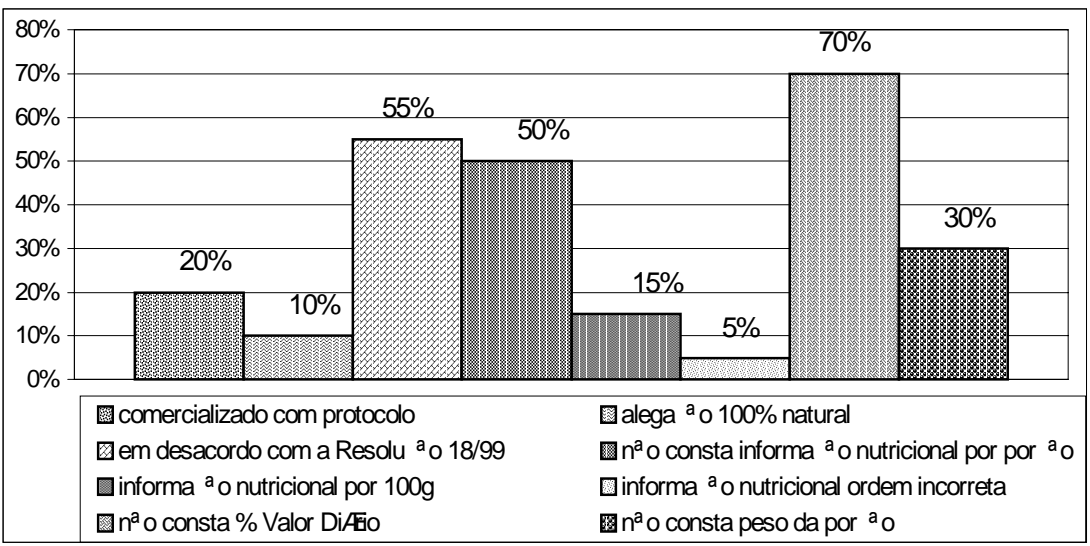


Nenhuma irregularidade foi encontrada nos rótulos dos produtos enquadrados nas Portarias n.34/98 (alimentos de transição para lactentes e crianças de primeira infância) 4 rótulos, 977/98 (fórmulas infantis para lactentes) 6 rótulos e 223/98 (complementos alimentares para gestantes e nutrizes) 1 rótulo $(14,17)$.

\title{
4 CONCLUSÃO
}

A maioria dos rótulos dos produtos alimentícios comercializados apresentou irregularidades em relação à legislação vigente, incluindo ausência de declarações e/ou de advertências obrigatórias (que deveriam aparecer em destaque ou em negrito). A aquisição de produtos influenciada por estratégias de mercado, acrescida pelas irregularidades apontadas pode causar danos à saúde do consumidor e prejuízos econômicos.

Constatou-se pouco compromisso ético de algumas empresas para com o consumidor, ocasionando irregularidades que vão desde a falta de informação à informação enganosa ou inadequada relativas aos produtos comercializados. As constantes modificações na legislação aliadas à fiscalização precária são outros fatores que contribuem para essa situação.

Verificou-se a necessidade de abordar aspectos de rotulagem de alimentos em programas de educação nutricional para conscientizar o consumidor a respeito das informações que devem ser fornecidas pelo fabricante.

\begin{abstract}
FOOD LABELING AS A MEANS OF INFORMATION TO THE CONSUMER: ADEQUACY AND IRREGULARITIES

It was investigated for adequacy 220 food labels of different categories, with emphasis on food for special dietary uses and foods with essential nutrients added. It was observed that most of the manufacturers do not fulfill the actual Brazilian legislation because all the labels analyzed showed some irregularities. It was verified absence of information about the product conservation (25\%) and the presence of colorant or artificial flavor $(16,77 \%)$ in the foods. Also information that could confuse the consumer $(19,9 \%)$ was found, including allegations of therapeutic properties of the food. In more than $50 \%$ of the labels analyzed according to Resolution RDC 40/01, a discrepancy involving the established nutritional information was verified. The main inconsistencies in relation to the actual law involved obligatory information and statements of each norm. It was concluded that most of the labels in the market was in disagreement with the law, proclaiming the need of a more efficient control and a higher commitment of the manufacturers with the consumer.
\end{abstract}

KEY-WORDS: FOOD-LABELING; FOOD-REGULATIONS. 


\section{REFERÊNCIAS}

1 BRASIL. Ministério da Justiça. Secretaria do Direito Econômico. Lei 3078 de 11 de setembro de 1990. Código de Defesa do Consumidor. Disponível em <www.mj.gov.br/dpdc/cdc.htm>. Acesso em 12/03/03.

2 BRASIL. Ministério da Saúde. Agência Nacional de Vigilância Sanitária. Resolução RDC ANVISA/MS $n^{\circ} \mathbf{2 5 9}$, de 20 de setembro de 2002. Regulamento técnico para rotulagem de alimentos embalados. Disponível em <www.anvisa.gov.br>. Acesso em 12/03/03.

3 BRASIL. Ministério da Saúde. Secretaria de Vigilância Sanitária. Portaria SVS no 42 de 14 de janeiro de 1998. Regulamento técnico para rotulagem nutricional de alimentos embalados. Disponível em: <www.anvisa.gov.br>. Acesso em 15/08/ 2002.

BRASIL. Ministério da Saúde. Agência Nacional de Vigilância Sanitária. Resolução RDC n$^{\circ} \mathbf{4 0}$ de $\mathbf{2 1}$ de março de 2001. Regulamento técnico para rotulagem nutricional obrigatória de alimentos e bebidas embalados. Disponível em: $<$ www.anvisa.gov.br>. Acesso em 12/03/03.

5 BRASIL. Ministério da Saúde. Secretaria de Vigilância Sanitária. Portaria SVS no 27 de 13 de janeiro de 1998. Regulamento técnico referente à informação nutricional complementar. Disponível em: <www.anvisa.gov.br>. Acesso em 12/ 03/03.

6 BRASIL. Ministério da Saúde. Secretaria de Vigilância Sanitária. Portaria SVS no 29 de 13 de janeiro de 1998. Regulamento técnico para fixação de identidade e qualidade de alimentos para fins especiais. Disponível em: <www.anvisa.gov.br>. Acesso em 12/03/03.

BRASIL. Ministério da Saúde. Secretaria de Vigilância Sanitária. Portaria SVS no 30 de 13 de janeiro de 1998. Regulamento técnico para fixação de identidade e qualidade de alimentos para controle de peso. Disponível em: <www.anvisa.gov.br>. Acesso em 12/03/03.

8 BRASIL. Ministério da Saúde. Secretaria de Vigilância Sanitária. Portaria SVS no 31 de 13 de janeiro de 1998. Regulamento técnico para fixação de identidade e qualidade de alimentos adicionados de nutrientes essenciais. Disponível em: <www.anvisa.gov.br>. Acesso em 12/03/03.

BRASIL. Ministério da Saúde. Secretaria de Vigilância Sanitária. Portaria SVS no 32 de 13 de janeiro de 1998. Regulamento técnico para fixação de identidade e qualidade de suplementos vitamínicos e/ou de minerais. Disponível em: <www.anvisa.gov.br>. Acesso em 12/03/03.

10 BRASIL. Ministério da Saúde. Secretaria de Vigilância Sanitária. Portaria SVS no 38 de 13 de janeiro de 1998. Regulamento técnico para fixação de identidade e qualidade de adoçantes de mesa. Disponível em: <www.anvisa.gov.br>. Acesso em $12 / 03 / 03$. 
54 de 04 de julho de 1995. Padrão de identidade e qualidade para sal hipossódico. Disponível em: <www.anvisa.gov.br>. Acesso em 12/03/03.

12 BRASIL. Ministério da Saúde. Secretaria de Vigilância Sanitária. Portaria SVS no 222 de 24 de março de 1998. Regulamento técnico para fixação de identidade e qualidade de alimentos para praticantes de atividade física. Disponível em: $<$ www.anvisa.gov.br>. Acesso em 12/03/03.

13 BRASIL. Ministério da Saúde. Agência Nacional de Vigilância Sanitária. Portaria ANVS no 449 de 09 de setembro de 1999. Aprova o Regulamento técnico referente a alimentos para nutrição enteral. Disponível em: <www.anvisa.gov.br>. Acesso em 12/03/03.

14 BRASIL. Ministério da Saúde. Secretaria de Vigilância Sanitária. Portaria SVS no 34 de 13 de janeiro de 1998. Regulamento técnico para fixação de identidade e qualidade de alimentos de transição para lactentes e crianças de primeira infância. Disponível em: <www.anvisa.gov.br>. Acesso em 12/03/03.

15 BRASIL. Ministério da Saúde. Secretaria de Vigilância Sanitária. Portaria SVS no 36 de 13 de janeiro de 1998. Regulamento técnico para fixação de identidade e qualidade de alimentos à base de cereais para alimentação infantil. Disponível em: <www.anvisa.gov.br>. Acesso em 12/03/03.

16 BRASIL. Ministério da Saúde. Secretaria de Vigilância Sanitária. Portaria SVS no 977 de 05 de dezembro de 1998. Regulamento técnico para fixação de identidade e qualidade de fórmulas infantis para lactentes. Disponível em: $<$ www.anvisa.gov.br>. Acesso em 12/03/03.

17 BRASIL. Ministério da Saúde. Secretaria de Vigilância Sanitária. Portaria SVS no 223 de 24 de março de 1998. Regulamento técnico para fixação de identidade e qualidade de complementos alimentares para gestantes ou nutrizes. Disponível em: <www.anvisa.gov.br>. Acesso em 12/03/03.

18 BRASIL. Ministério da Saúde. Agência Nacional de Vigilância Sanitária. Resolução RDC 222 de 05 de agosto de 2002. Regulamento técnico para promoção comercial de alimentos para lactentes e crianças de primeira infância. Disponível em: <www.anvisa.gov.br>. Acesso em 12/03/03.

19 BRASIL. Ministério da Saúde. Agência Nacional de Vigilância Sanitária. Resolução ANVS no 16 de 03 de dezembro de 1999. Procedimentos para registro de alimentos e ou novos ingredientes. Disponível em: <www.anvisa.gov.br>. Acesso em 12/03/03.

20 BRASIL. Ministério da Saúde. Agência Nacional de Vigilância Sanitária. Resolução ANVS no 18 de 03 de dezembro de 1999. Regulamento técnico que estabelece as diretrizes básicas para análise e comprovação de propriedades funcionais e ou de saúde alegadas em rotulagem de alimentos. Disponível em: $<$ www.anvisa.gov.br>. Acesso em 12/03/03.

21 CÂNDIDO, Lys Mary Bileski. Atualização da legislação de alimentos. Revista Higiene Alimentar, São Paulo, v. 14, n. 78/79, p. 103-112, 2000. 